\title{
Imaging the molecular dynamics of dissociative electron attachment to water
}

\author{
H. Adaniya, ${ }^{1,2}$ B. Rudek, ${ }^{2,3}$ T. Osipov, ${ }^{2}$ D. J. Haxton, ${ }^{2}$ \\ T. Weber, ${ }^{2}$ T. N. Rescigno, ${ }^{2}$ C. W. McCurdy, ${ }^{2,4}$ and A. Belkacem ${ }^{2}$ \\ ${ }^{1}$ Department of Applied Science, University of California, Davis, CA 95616, USA \\ ${ }^{2}$ Lawrence Berkeley National Laboratory, Chemical Sciences, Berkeley, CA 94720, USA \\ ${ }^{3}$ J. W. Goethe Universität, 60438 Frankfurt, Germany \\ ${ }^{4}$ Departments of Applied Science and Chemistry, University of California, Davis, CA 95616, USA
}

\begin{abstract}
Momentum imaging experiments on dissociative electron attachment to the water molecule are combined with $a b$ initio theoretical calculations of the angular dependence of the quantum mechanical amplitude for electron attachment to provide a detailed picture of the molecular dynamics of dissociation attachment via the two lowest energy Feshbach resonances. The combination of momentum imaging experiments and theory can reveal dissociation dynamics for which the axial recoil approximation breaks down and thus provides a powerful reaction microscope for DEA to polyatomics.
\end{abstract}

PACS numbers: $34.80 . \mathrm{Ht}$

Resonant collisions between low-energy electrons and molecules can provide an efficient pathway for channeling electronic energy into nuclear motion. In dissociative electron attachment (DEA), the transient negative ions so formed fragment to form neutral plus ionic fragments. The resurgence of interest in this process in recent years has been due in large part to the key role it plays in radiation damage in a number of different contexts and to the discovery that low-energy DEA can be responsible for double-strand breaks in DNA [1]. It is therefore not surprising that dissociative electron attachment to water has been the target of much recent experimental and theoretical work, since water is the principal constituent is living tissue and DEA can produce free radicals that affect that tissue. The application of modern imaging techniques, such as velocity slice imaging [2], can bring a new level of sophistication to the study of the angular dependence of fragment ions produced in DEA.

Dissociative electronic attachment to the deceptively simple water molecule involves complex electronic and nuclear dynamics. In the gas phase, it proceeds via three transient anion states of ${ }^{2} \mathrm{~B}_{1},{ }^{2} \mathrm{~A}_{1}$ and ${ }^{2} \mathrm{~B}_{2}$ symmetries which are responsible for three distinct broad peaks in the DEA cross section at electron energies of $6.5,9$ and $12 \mathrm{eV}$ [4], while in the condensed phase, there is evidence that deep-valence states may be responsible for a broad DEA peak centered at $25 \mathrm{eV}$ [3]. The negative ion states subsequently fragment to produce the anions $\mathrm{H}^{-}, \mathrm{O}^{-}$and possibly $\mathrm{OH}^{-}$, in various two-body as well as three-body breakup channels [5-9]. In this Letter we present momentum imaging measurements of the angular distribution of the ionic fragments relative to the direction of the incident electron that allow us to probe those dynamics. However, since the measurements are necessarily made in the laboratory frame, these observations can yield detailed information about the nuclear dynamics following electron attachment only if a reliable connection between the lab frame and molecular frame can be made. The key to that connection is a knowledge of the angular dependence of the electron attachment probability in the molecular frame, and that attachment probability can be calculated by $a b$ initio methods [9-12]. The attachment probability can be directly related to the laboratory frame distribution when the axial recoil condition is met, requiring in the present context that the recoil axis which connects the atom and the diatom center of mass does not rotate during the dissociation. We demonstrate here that this combination of momentum imaging experiments and theory can also reveal dissociation dynamics for which the axial recoil approximation breaks down and thus provides a powerful reaction microscope for DEA to polyatomics.

In our experiments, ions resulting from DEA are extracted by means of a COLTRIMS-like spectrometer [13]. The time-of-flight and detector positions of the ions allow for reconstruction of their three-dimensional momentum vectors. The electron-scattering calculations that provide the connection between lab and molecular frames employ the well-established complex Kohn variational method [10], and are augmented by classical trajectory calculations performed on previously constructed potential energy surfaces [11] to allow comparison with experiment.

To understand the relation of lab- to body-frame dynamics it is useful to examine the classical formula [14] that describes the angular distribution, $I\left(\theta_{L}, \phi_{L}\right)$, of two fragments in the lab frame following electron attachment in which the electron is incident along the $\mathrm{z}$ axis or following photoabsorption with polarization along that axis,

$$
I\left(\theta_{L}, \phi_{L}\right)=\int d \Omega P(\alpha, \beta, \gamma) f\left(\theta_{m}, \phi_{m}\right)
$$

where $P(\alpha, \beta, \gamma)$ is the probability of a molecule's attaching an electron or absorbing a photon in the orientation specified by the Euler angles $\alpha, \beta, \gamma$ connecting 

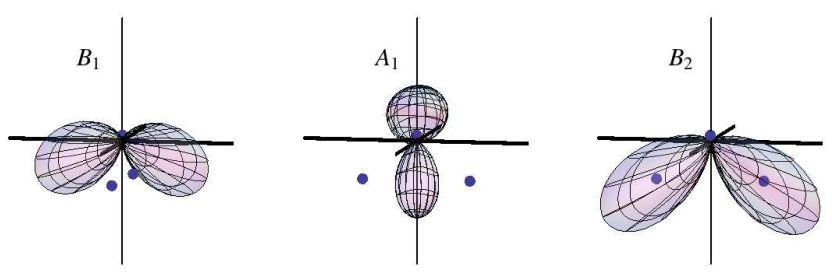

FIG. 1: (Color online) Electron attachment probability calculated at the equilibrium geometry of the neutral water molecule for the three resonances as a function of polar angles about the center of mass. Dots denote positions of nuclei.

the molecular and lab frames, and $f\left(\theta_{m}, \phi_{m}\right)$ is the distribution of the two fragments in the molecular frame after dissociation. The integral $d \Omega$ is over the Euler angles. Equation (1) is the textbook expression [14] used to describe the angular dependence of photodissociation. The key difference between the two processes is that in the case of photodissociation $P(\alpha, \beta, \gamma)$ is proportional to $\cos ^{2} \beta$ (the square of a dipole amplitude), and the integration over molecular orientations (equivalent to treating rotation classically) gives only the familiar formula $I \propto 1+\beta(E) P_{2}\left(\cos \theta_{L}\right)$, where $P_{2}$ is a Legendre polynomial and $\beta(E)$ is the asymmetry parameter.

In DEA the role of the dipole amplitude is played instead by the square of a quantity called the entrance amplitude [15], $V_{\bar{a}}(\theta, \phi)$, which depends on two angles instead of one as in photodissociation. This function corresponds to the matrix element between a discrete resonance state and a background scattering wavefunction for an electron incident on the initial target state,

$$
V_{\bar{a}}(\theta, \phi ; \vec{R})=\left\langle\Psi_{\mathrm{bg}}^{+}(\theta, \phi ; \vec{R})\left|H_{e l}\right| \Psi^{\mathrm{res}}(\vec{R})\right\rangle .
$$

Here $\Psi_{b q}^{+}(\theta, \phi)$ is the background function with a plane wave incident on the target in direction $\theta, \phi, \Psi^{\text {res }}$ is a discrete-state approximation to the resonance wavefunction, and $H_{e l}$ is the electronic part of the Hamiltonian. The entrance amplitude can also be understood in terms of the residue of the fixed-nuclei S-matrix at the complex resonance energy $[10,11]$.

Fig. 1 shows the calculated entrance probabilities for the $\left(1 \mathrm{~b}_{1}^{-1} 4 \mathrm{a}_{1}^{2}\right)^{2} \mathrm{~B}_{1},\left(3 \mathrm{a}_{1}^{-1} 4 \mathrm{a}_{1}^{2}\right)^{2} \mathrm{~A}_{1}$ and $\left(1 \mathrm{~b}_{2}^{-1} 4 \mathrm{a}_{1}^{2}\right)^{2} \mathrm{~B}_{2}$ resonances. The entrance amplitude reflects the underlying shape of the $1 b_{1}, 1 a_{1}$ and $1 b_{2}$ orbitals of neutral water, from which one electron is excited into the $4 \mathrm{a}_{1}$ orbital to form the ${ }^{2} \mathrm{~B}_{1},{ }^{2} \mathrm{~A}_{1}$ and ${ }^{2} \mathrm{~B}_{2}$, Feshbach resonance configurations, respectively [4]. For the ${ }^{2} \mathrm{~B}_{1}$ resonance the electron is more likely to attach when the electron impinges on the molecule off the plane formed by the oxygen and the two hydrogen atoms. For the ${ }^{2} \mathrm{~A}_{1}$ resonance the electron is more likely to attach along the main axis of symmetry of the molecule, while for the ${ }^{2} \mathrm{~B}_{2}$ resonance the electron is more likely to attach when impinging along either $\mathrm{O}-\mathrm{H}$ bond. The more complicated dependence of the attachment dependence on the angle of incidence of the electron provides a stronger connection between the molecular and laboratory frames than in photodissociation that yields a more complicated angular dependence of the fragments in general and thus more information on the molecular dynamics of dissociation in DEA than is available in photodissociation. We focus here on the ${ }^{2} \mathrm{~B}_{1}$ and ${ }^{2} \mathrm{~A}_{1}$ resonances, deferring analysis of the ${ }^{2} \mathrm{~B}_{2}$ resonance to a later paper.

We study interactions between water molecules and electrons by colliding a magnetically collimated and pulsed electron beam at $90^{\circ}$ with a beam of water molecules from an effusive source. In a COLTRIMSlike spectrometer [13] we measured the ions' time-of-flight and position at the detector following fragmentation to reconstruct their momentum vectors in $3 \mathrm{D}$. The electron beam is collimated by a magnetic field of $\approx 25$ Gauss, created by a pair of Helmholtz coils. The coils are air cooled to prevent a change in the strength of the magnetic field. A pulsed electron beam with $80 \mathrm{~ns}$ pulse width at 50 $\mathrm{kHz}$ repetition rate is generated by a Kimball Physics ELG-2A electron gun. A surgical needle of $0.5 \mathrm{~mm}$ internal diameter is used to produce an effusive jet of target water molecules. The needle and water vapor inlet tube inside the vacuum chamber are heated to prevent condensation and clustering. The chamber pressure at the interaction region is kept at $\approx 10^{-7}$ Torr with the gas water inlet closed and at $5 \times 10^{-6}$ Torr with the water inlet open. The difference in the background is mostly from water vapor.

Reactions take place between a pusher plate and a permanently grounded plate with a 1-inch diameter grid. The pusher plate voltage is turned on $\approx 50 \mathrm{~ns}$ after the electron beam pulse passes the interaction region and is kept on for about $10 \mu \mathrm{s}$. The field duration and its delay with respect to the pulsed electron beam are set to optimize the quality of the electronic signal. The grounded plate with grid is part of a large cylindrical enclosure that covers the rest of the parallel plate spectrometer together with a RoentDek 2D delay line detector. It minimizes the chances of stray electrons hitting the detector and also shields the detectors from the electronic noise associated with the pulsed pusher field. Negative ions are extracted by a pusher field of $25 \mathrm{~V} / \mathrm{cm}$ into the shielded part of the spectrometer. Ions are accelerated by a uniform electric field and focused by an electrostatic lens, created by a series of copper plates, to minimize the effect of the reaction position defined by the effusive target and expansion of the anion momentum sphere prior to extraction. This set-up allows for $4 \pi$ solid angle detection of ions with energies between 0 and $10 \mathrm{eV}$. Reconstructed 3D momentum vectors provide information about the kinetic energy release (KER) and the angular distribution of the negative fragments with respect to the incidence angle of the electron beam. Details of the set-up and spectrometer 

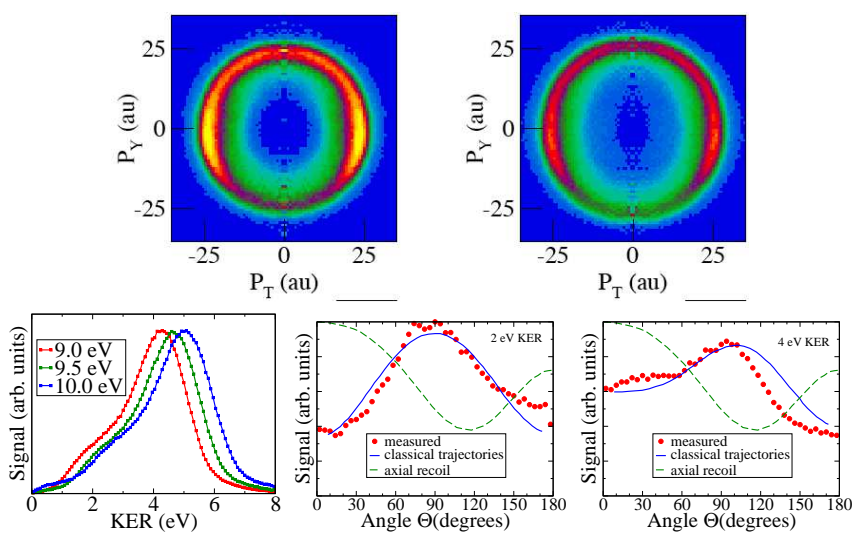

FIG. 2: (Color online) Top: Scatter plots for $\mathrm{H}^{-}$production from ${ }^{2} \mathrm{~A}_{1}$ resonance at 9.0 and $10 \mathrm{eV}$. Bottom: angular integrated experimental KER distributions(left); comparison of measured and calculated angular distributions at $2 \mathrm{eV}$ (middle) and $4 \mathrm{eV}$ (right) at $9.5 \mathrm{eV}$.

will be presented in a separate publication.

${ }^{2} \mathbf{B}_{1}$ resonance: Dissociation through the $\mathrm{H}^{-}+\mathrm{OH}$ channel is well known to be dominant for this resonance and our results for this channel are in accord with prior results $[5-8,16]$. We find that the angular distribution of $\mathrm{H}^{-}$with respect to the incident electron peaks at about $100^{\circ}$, in agreement with prior results [6]. This channel is accurately described by assuming the axial recoil approximation, as we have found previously [10].

The conclusions drawn from our current measurements on DEA through ${ }^{2} \mathrm{~B}_{1}$ differ from earlier studies with respect to the minor $\mathrm{O}^{-}+\mathrm{H}_{2}$ channel. Previous experiments $[5,8]$ have reported $\mathrm{O}^{-}$from ${ }^{2} \mathrm{~B}_{1}$ with a peak intensity approximately one fortieth that of $\mathrm{H}^{-}$. However, full polyatomic calculations on this system [12] failed to reproduce this result, instead predicting $\mathrm{O}^{-} / \mathrm{H}^{-}$ratios of $\sim 0.001$. We have taken care to separate the $\mathrm{O}^{-}$ signals that come from dissociative attachment to water from other background sources. We conclude that most of the $\mathrm{O}^{-}$observed and reported in the literature comes from electron attachment to the residual $\mathrm{O}_{2}$ background in the vacuum chamber despite the fact that the vacuum is held at $10^{-7}$ Torr. The $\mathrm{O}^{-}$signal that results following subtraction of the background is about three orders of magnitude smaller than $\mathrm{H}^{-}$production. A more detailed discussion of this important observation will be presented in a future publication.

${ }^{2} \mathbf{A}_{1}$ resonance: In agreement with prior results [58], $\mathrm{H}^{-}$production from the ${ }^{2} \mathrm{~A}_{1}$ resonance is found to be approximately an order of magnitude larger than $\mathrm{O}^{-}$ production. Our results on $\mathrm{H}^{-}$are shown in Fig. 2. The $\mathrm{H}^{-}$kinetic energy distributions, shown in the bottomleft panel, shift to higher energy with increasing electron energy, but, unlike the case with the ${ }^{2} \mathrm{~B}_{1}$ resonance, the width of the distributions do not change with energy, ie., most of the increased electronic energy is converted into translational energy of the fragments with little change of the vibrational and rotational energies of the $\mathrm{OH}$ fragment. An interesting feature is that the kinetic energy release exhibits a marked shoulder on the left side of the distribution. The portion of the distribution to the left of the shoulder contributes $\sim 15 \%$ of the total distribution independent of incident energy. On energetic grounds, we can rule out any contribution arising from production of electronically excited $\mathrm{OH}\left({ }^{2} \Sigma\right)$ or from three-body breakup, which means that the low kinetic energy peak corresponds to an $\mathrm{OH}$ fragment left in its ground electronic state with high rovibrational energy.

The angular distributions of the $\mathrm{H}^{-}$fragment, shown in the bottom-middle and bottom-right panels of Fig. 2 for low and high KER, are far less peaked than they are in the case of the ${ }^{2} \mathrm{~B}_{1}$ resonance. Those at low and high KER are different. The angular dependence itself depends upon the final state of the $\mathrm{OH}$ fragment. Therefore, the axial recoil approximation fails for DEA to the ${ }^{2} \mathrm{~A}_{1}$ state.

To understand these experimental results, we carried out classical trajectory calculations using the previously constructed [11] potential energy surface for the $1^{2} \mathrm{~A}^{\prime}$ state. We find that the $\mathrm{HOH}$ bond angle opens up quickly following attachment, confirming that the condition of axial recoil is broken. Furthermore, the degree of opening is greatest when the molecule dissociates symmetrically along both $\mathrm{OH}$ bonds.

In Fig. 2 we compare the experimental results with numerical predictions at two different levels of theory. At the simplest level (dashed lines in Fig. 2), we use the attachment probability along with the axial recoil assumption to produce an angular distribution prediction that is independent of KER and that does not account for the dissociation dynamics. We also show results (solid lines) of a more elaborate treatment in which we convolve the attachment probability predictions with the recoil angle distributions for different KERs obtained from the classical trajectory calculations. This treatment also includes the variation of the attachment probability with geometry and clearly gives a good match with the experimental results. These calculations indicate that the observed angular distribution is a product of multiple dynamic effects, including the variation of entrance amplitude with nuclear geometry and the correlation between the geometry at attachment and the angle and kinetic energy of $\mathrm{H}^{-}$recoil.

$\mathrm{O}^{-}$production through the ${ }^{2} \mathrm{~A}_{1}$ state appears to proceed predominantly through a three-body breakup, $\mathrm{H}+$ $\mathrm{H}+\mathrm{O}^{-}$, as we recently predicted [9]. This breakup channel was previously observed for $\mathrm{D}_{2} \mathrm{O}$ [17]. Fig. 3 shows the measured angular distributions of $\mathrm{O}^{-}$along with the theoretical prediction assuming axial recoil and the attachment probability shown in Fig. 1. The momentum spheres (longitudinal versus transverse momen- 

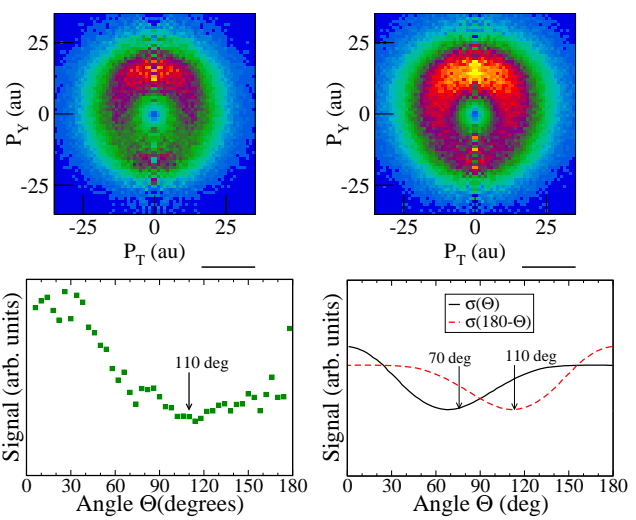

FIG. 3: (Color online) Top: Scatter plots for $\mathrm{O}^{-}$production from ${ }^{2} \mathrm{~A}_{1}$ resonance at 9.5 and $10 \mathrm{eV}$. Bottom: measured angular distributions at $9.5 \mathrm{eV}$ (left), compared with theoretical predictions (right), the latter plotted as functions of $\Theta$ and $180-\Theta$ (see text).

tum) shown in the top panels in Fig. 3 all exhibit an intense and broad $\mathrm{O}^{-}$distribution in the forward direction and a less intense and narrow distribution in the backward direction for three electron energies across the ${ }^{2} \mathrm{~A}_{1}$ resonance. The bottom panels of the figure show the measured and predicted angular distributions for $9.5 \mathrm{eV}$ electron energy. It is clear that the measured distribution and the theoretical prediction appear to be mirror images of each other, the measured angular distribution showing a broad minimum about $110^{\circ}$ while the theoretical minimum assuming axial recoil is located at $180^{\circ}$ $110^{\circ}=70^{\circ}$. The contrast between the measured distribution and the axial recoil prediction suggests that after attachment, the molecule scissors backwards and ejects the oxygen through the mouth of the $\mathrm{H}-\mathrm{O}-\mathrm{H}$ bond, regardless of the direction of the incident electron, while the hydrogens recoil in the opposite direction. This interpretation of the dissociation dynamics, which is also supported by classical trajectory calculations, explains why $\mathrm{O}^{-}$production through the ${ }^{2} \mathrm{~A}_{1}$ state is most likely to proceed through three-body breakup and why the associated angular distribution is the inverse of the axial recoil prediction.

We have shown that a calculation of the attachment probability, which ties the molecular- to the laboratoryframe, and a COLTRIMS-like experimental momentum imaging technique can be combined to create a unique reaction microscope that provides insight into the dynamics involved in dissociative electron attachment. The power of the method was illustrated by application of this reaction microscope to the case of DEA in water where there was existing extensive $a b$ initio theoretical work. The power of this new approach can now be directly applied to more complex molecules were the entrance amplitude can be calculated reasonably accurately.

This work was performed under the auspices of the U.S. Department of Energy by LBNL under contract No. DE-AC02-05CH11231 and was supported by the DOE OBES, Division of Chemical Science.

[1] B. Boudaïffa, P. Cloutier, D. Hunting, M. A. Huels, and L. Sanche, Science 287, 1658 (2000).

[2] D. Nandi, V. S. Prabhudesai, E. Krishnakumar, and A. Chatterjee, Rev. Scien. Instr. 76, 053107 (2005).

[3] G. A. Kimmel and T. M.. Orlando, Phys. Rev. Lett. 77, 3983 (1996).

[4] M. Jungen, J. Vogt, and V. Staemmler, Chem. Phys. 37, 49 (1979).

[5] J. Fedor, P. Cicman, B. Coupier, S. Feil, M. Winkler, K. Gluch, J. Husarik, D. Jaksch, B. Farizon, N. J. Mason, P. Scheier and T. D. Märk, J. Phys. B 39, 3935 (2006).

[6] D. S. Belic, M. Landau, and R. H. Hall, J. Phys. B. 14, 175 (1981).

[7] R. N. Compton and L. G. Christophorou, Phys. Rev. 154, 110 (1967).

[8] C. E. Melton, J. Chem. Phys. 57, 4218 (1972).

[9] D. J. Haxton, T. Rescigno, and C. W. McCurdy, Phys. Rev. A 78, 040702 (2008).

[10] D. J. Haxton, C. W. McCurdy, and T. N. Rescigno, Phys. Rev. A 73, 062724 (2006).

[11] D. J. Haxton, C. W. McCurdy, and T. N. Rescigno, Phys. Rev. A 75, 012710 (2007).

[12] D. J. Haxton, T. N. Rescigno, and C. W. McCurdy, Phys. Rev. A 75, 012711 (2007).

[13] R. Dörner, et al., Phys. Rep. 330, 96 (2000).

[14] R. N. Zare, Angular Momentum: Understanding Spatial Aspects in Chemistry and Physics (John Wiley \& Sons, 1988).

[15] T. F. O’Malley, Phys. Rev. 150, 14 (1966).

[16] N. B. Ram, V. S. Prabhudesai, G. Aravind, P. Rawat, and E. Krishnakumar, Journal of Physics: Conf. Series 80, 012017 (2007).

[17] M. G. Curtis and I. C. Walker, J. Chem. Soc. Faraday Trans. 88, 2805 (1992). 